\title{
The Expansion of the Universe
}

\section{Dr. Andreas Gimsa}

Teaching Stirling Technology Institute gemeinnützige GmbH D-14478 Potsdam, Germany

\section{Abstract}

The expansion of the universe is explained, calculated and graphically displayed. The $3 \mathrm{~K}$ background radiation is examined and interpreted as reflected and distributed stellar radiation. The role of entropy in cosmology is discussed. In our expanding universe it must remain constant. Physical quantities previously assumed to be constant are worked out to be variable. It is explained why the measured redshift is not due to an accelerated growth of the universe.

Keywords: Space expansion, Entropy, Redshift, Background radiation.

\section{Introduction}

Today's expansion theory of the universe is based on a big bang, which is said to have accelerated a singular point to its present mass and size. Immediately after the Big Bang, an inflationary phase of expansion is said to have taken place in the age range from $10^{-35} s$ to about $10^{-33} s$ (possibly also up to $10^{-30} s$ ), during which the universe is said to have expanded by the factor $10^{26}$. The author is convinced that these ideas are incorrect and should be overcome.

The universe represents a spherical, adiabatic, enclosed system. No energy and material can be transported across its system boundaries, because it expands exactly at the speed of light. To avoid misunderstandings, a clear distinction is made here between the speed of an object in space, which must be less than the speed of light, and the speed of space at the speed of light. What is meant here is that the ratio of the radius of the sphere and the age of the universe corresponds exactly to the speed of light. The expansion of the universe therefore takes place in an isentropic process. The isentropic exponent $\kappa$ must have the value $\kappa=4 / 3$ [1.].

\section{The 3K-background radiation}

It is to be mathematically proven that the $2.73 \mathrm{~K}$ background radiation is stellar radiation, which is distributed on the inner surface of the universe and is absorbed and reflected. It must remain trapped in an eternally pulsating universe. The background radiation forms the perfect profile of a blackbody in spherical space. The radius of space is $1.310^{26}$ meters at the current space age of $13.810^{9}$ years when the universe expands at the speed of light. With the assumption that the average star temperature today is like that of the Sun with 5,700 Kelvin, the radiation law of Stefan and Boltzmann allows the calculation of a theoretical spherical radius whose radiating surface is as large as the sum of all radiating star surfaces of the Universe [2.]

$$
r_{\text {uni }}^{2} \mathcal{T}_{\text {bg }}^{4}=r_{\text {big star }}{ }^{2} \mathcal{T}_{\text {star }}{ }^{4}
$$

The radius $r_{\text {big star }}$ of this theoretical spherical star according to equation (01) is $2.9910^{19} \mathrm{~m}$. If we now calculate its radiation surface and then put it in relation to that of a typical star, we arrive at the approximate number $N_{\text {star }}$ of stars in the universe. This typical star is supposed to be our Sun:

$$
N_{\text {star }}=\frac{A_{\text {bigstar }}}{A_{\text {star }}}=\frac{r_{\text {big star }}{ }^{2}}{r_{\text {star }}{ }^{2}}=\left(\frac{2,9910^{19}}{6,9610^{8}}\right)^{2}=1,8510^{21} \text { Sterne }
$$

If we assume that there are 100 billion stars per galaxy and 10 billion galaxies, we arrive at a number of $N_{\text {star }}=10^{21}$ stars. Here there are different data in the literature about the observations, but they are of a similar order of magnitude. 
The calculated number of stars according to equation (02) therefore agrees quite well with the observed number. The stellar radiation could therefore actually be in radiative equilibrium with the surface (the horizon) of the universe and lead to background radiation.

\section{The expansion of the universe graphically and mathematically}

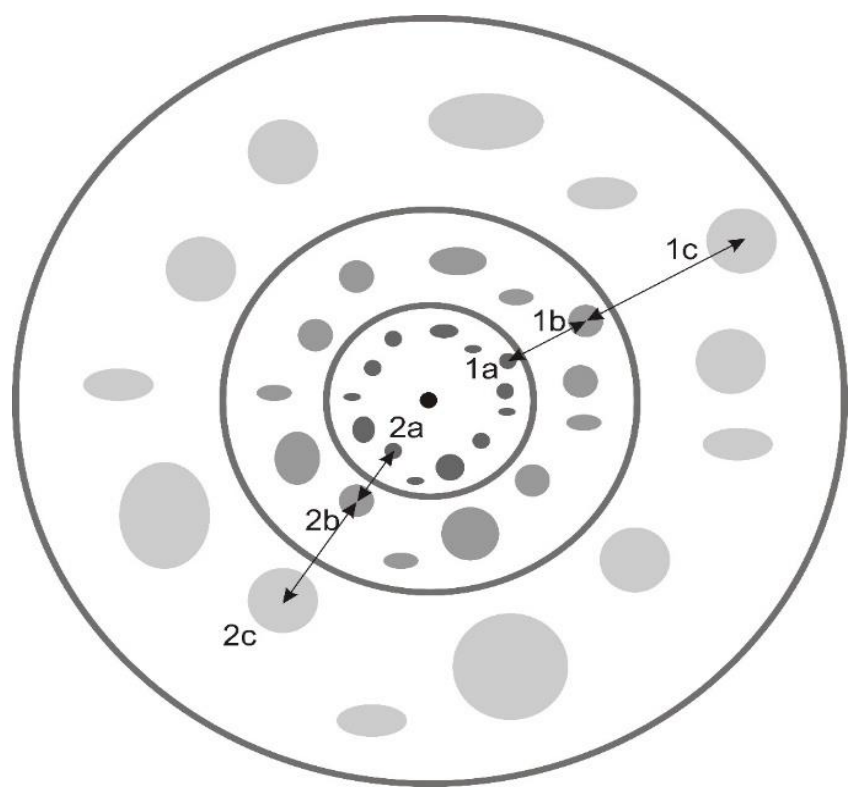

Figure 1: Expansion of the universe and galaxies in three age states (spherical shell regions)

Figure 1 illustrates the expansion in three age states. The first state is delimited by the first circumscribed circle, which is the first radius. Within this boundary the galaxies are the smallest and most massive. From the first to the second state, the radius of the universe has doubled. The radii of the galaxies are also twice as large and their mass has halved, just like that of the universe. From the second to the third state, the radius has doubled again. The galaxies have again become twice as large and their mass has halved again, just like that of the universe. How can the speeds of the galaxies be described? All speeds refer to the center of the universe. The radius of the universe increases at the speed of light. This means that because the universe is $13.810^{9}$ years old, it is now $1.310^{26}$ meters. Galaxy 1 in Figure 1 is somewhat slower. It travels a smaller distance (distance 1a-1b) in the time it takes for the radius to double. Its speed with space is therefore less than the speed of light. Galaxy 2, which lies further in the universe, is even slower than galaxy 1. Its path in the same time is even smaller (distance 2a-2b). However, the paths of all galaxies double in the same way as the radius of the universe doubles. Their respective speeds with space maintain the same size.

A galaxy that would be exactly in the center of the Universe would have the velocity $v=0$ with space. It would merely expand in all directions with age. All distance ratios remain the same as the universe expands, e.g. the ratio of distances $(2 c-2 b) /(2 b-2 a)=(1 c-1 b) /(1 b-1 a)$.

As space and objects expand, the distances between galaxies increase in proportion to the radius of space, not only in radial directions but also in any other direction in space: if the radius of space doubles, so do the distances (Figure 1).

One can speak of an expansion in which the galaxies maintain their different velocities. As everything gets older, all radii and distances become larger. All scales grow with the universe.

Our galaxy does not occupy a preferential place in the universe. One could theoretically divide the Universe into two spherical shell regions and define a spherical parting plane at a distance of half the radius from the center. If the arrangement of galaxies is evenly distributed in space, what would be the probability that our galaxy is located in the outer spherical shell? The probability is proportional to the volume of space. According to this, it is 7 times more likely that we are in the outer and not the inner part of the universe. The probability of being on the outside is $87.5 \%$. 


\begin{tabular}{|c|c|c|c|c|c|c|c|c|}
\hline $\mathbf{S}_{\text {tep }}$ & $\mathbf{t}_{\text {uni }}$ & $\mathbf{r}_{\text {uni }}$ & $\mathbf{v}_{\text {uni }}$ & $\mathbf{r}_{\text {bigstar }}$ & $\mathbf{m}_{\text {uni }}$ & $\mathbf{E}_{\text {uni }}$ & $\mathbf{v}_{\text {uni }}$ & $\rho_{\text {uni }}$ \\
\hline 1. & $8.500 \mathrm{E}+14$ & $2.548 \mathrm{E}+23$ & $2.998 \mathrm{E}+08$ & $5.845 \mathrm{E}+16$ & $8.997 \mathrm{E}+55$ & $8.086 \mathrm{E}+72$ & $6.931 \mathrm{E}+70$ & $1.298 \mathrm{E}-15$ \\
2. & $1.700 \mathrm{E}+15$ & $5.096 \mathrm{E}+23$ & $2.998 \mathrm{E}+08$ & $1.169 \mathrm{E}+17$ & $4.498 \mathrm{E}+55$ & $4.043 \mathrm{E}+72$ & $5.545 \mathrm{E}+71$ & $8.113 \mathrm{E}-17$ \\
\hline 3. & $3.400 \mathrm{E}+15$ & $1.019 \mathrm{E}+24$ & $2.998 \mathrm{E}+08$ & $2.338 \mathrm{E}+17$ & $2.249 \mathrm{E}+55$ & $2.022 \mathrm{E}+72$ & $4.436 \mathrm{E}+72$ & $5.071 \mathrm{E}-18$ \\
4. & $6.800 \mathrm{E}+15$ & $2.039 \mathrm{E}+24$ & $2.998 \mathrm{E}+08$ & $4.676 \mathrm{E}+17$ & $1.125 \mathrm{E}+55$ & $1.011 \mathrm{E}+72$ & $3.549 \mathrm{E}+73$ & $3.169 \mathrm{E}-19$ \\
5. & $1.360 \mathrm{E}+16$ & $4.077 \mathrm{E}+24$ & $2.998 \mathrm{E}+08$ & $9.353 \mathrm{E}+17$ & $5.623 \mathrm{E}+54$ & $5.054 \mathrm{E}+71$ & $2.839 \mathrm{E}+74$ & $1.981 \mathrm{E}-20$ \\
6. & $2.720 \mathrm{E}+16$ & $8.154 \mathrm{E}+24$ & $2.998 \mathrm{E}+08$ & $1.871 \mathrm{E}+18$ & $2.812 \mathrm{E}+54$ & $2.527 \mathrm{E}+71$ & $2.271 \mathrm{E}+75$ & $1.238 \mathrm{E}-21$ \\
\hline 7. & $5.440 \mathrm{E}+16$ & $1.631 \mathrm{E}+25$ & $2.998 \mathrm{E}+08$ & $3.741 \mathrm{E}+18$ & $1.406 \mathrm{E}+54$ & $1.263 \mathrm{E}+71$ & $1.817 \mathrm{E}+76$ & $7.737 \mathrm{E}-23$ \\
8. & $1.088 \mathrm{E}+17$ & $3.262 \mathrm{E}+25$ & $2.998 \mathrm{E}+08$ & $7.482 \mathrm{E}+18$ & $7.029 \mathrm{E}+53$ & $6.317 \mathrm{E}+70$ & $1.454 \mathrm{E}+77$ & $4.836 \mathrm{E}-24$ \\
\hline 9. & $2.176 \mathrm{E}+17$ & $6.523 \mathrm{E}+25$ & $2.998 \mathrm{E}+08$ & $1.496 \mathrm{E}+19$ & $3.514 \mathrm{E}+53$ & $3.159 \mathrm{E}+70$ & $1.163 \mathrm{E}+78$ & $3.022 \mathrm{E}-25$ \\
$\mathbf{1 0 .}$ & $\mathbf{4 . 3 5 2 \mathrm { E } + 1 7}$ & $\mathbf{1 . 3 0 5 E + 2 6}$ & $\mathbf{2 . 9 9 8 E + 0 8}$ & $\mathbf{2 . 9 9 3 E + 1 9}$ & $\mathbf{1 . 7 5 7 E}+53$ & $\mathbf{1 . 5 7 9 E}+70$ & $\mathbf{9 . 3 0 3 E}+78$ & $1.889 \mathrm{E}-26$ \\
\hline
\end{tabular}

\begin{tabular}{|c|c|c|c|c|c|c|c|c|}
\hline on & oigstar & $\mathcal{T}_{\text {bg }}$ & $\mathcal{T}_{\text {uni }}$ & $\gamma$ & $\mathcal{T}_{\text {uni }} V_{\text {uni }}$ & $\mathcal{T}_{\text {uni }} \mathbf{V}_{\text {uni }}{ }^{k-1}$ & & \\
\hline 1. & & & & & 030E-08 & & & \\
\hline 2. & & & & & & & & \\
\hline 3. & & & & & 8 & & & \\
\hline 4. & & & & & & & & \\
\hline 5. & & & & & 88 & & & \\
\hline 6. & & & & & & & & +9 \\
\hline 7. & & & & & & & & \\
\hline 8. & $280 t+04$ & & & & & & $=+90$ & 0 \\
\hline 9. & $140 E+04$ & & & & & & $E+98$ & $E+98$ \\
\hline 10 & $700 E+03$ & $730 E+00$ & $755 E-29$ & 11 & $.030 \mathrm{E}-08$ & -03 & $8.998 \mathrm{E}+98$ & $8.998 E+9$ \\
\hline
\end{tabular}

Table 1: Expansion of the universe with the development of important physical quantities

Table 1 illustrates the expansion of the universe in 10 age states by means of the evolution of important physical quantities. The age doubles from one state to the next until the 10th state is the age in space today. The table values are shown in SI units.

\section{Explanation of the table columns}

All physical quantities listed in the table are briefly discussed. In the first column is the age of the universe $t_{u n i}$. There are 10 age states shown. The beginning of the expansion is not shown in the first row. Instead, the first line 1/512 shows the current age, which is located in the last line.

In the second column is the radius of the universe. It results simply from the multiplication of the speed of light with the age: $r_{u n i}=c t_{\text {uni }}$.

The third column shows again explicitly the expansion of the universe with the speed of light in every age state: $v_{\text {uni }}=\frac{r_{u n i}}{t_{\text {uni }}}=c$.

The fourth column shows the radius $r_{\text {big star }}$ of a hypothetical superstar, which has the same surface area as all existing stars together at the respective age of the universe. The calculation is done for the last line (current age) according to equation (01). All other lines are proportional to the radius of the universe.

In the fifth column the mass of the universe is calculated. First the last line is calculated according to the formula $m_{u n i}=\frac{r_{u n i} c^{2}}{\gamma}$. All other lines can be calculated with the respective age of the universe, where $m_{\text {uni }} t_{\text {uni }}=$ const.

The sixth column shows the mass energy of the universe. It is $E_{u n i}=m_{u n i} c^{2}$ and decreases with increasing age.

The seventh column shows the volume of the universe, which is spherical: $V_{u n i}=\frac{4}{3} \pi r_{u n i}{ }^{3}$.

The eighth column represents the density of the universe: $\rho_{u n i}=\frac{m_{u n i}}{V_{u n i}}$. 
The ninth column shows the temperature $\mathcal{T}_{\text {big star }}$ of the hypothetical superstar. The last line is based on the temperature of the Sun. All other temperatures in the column are proportional to the mass of the universe.

In the tenth column the temperature $\mathcal{T}_{b g}$ of the background radiation is calculated. The last row is based on the current background radiation. Otherwise the temperature is proportional to $\mathcal{T}_{\text {big star }}$.

The previously unknown temperature of the universe $\mathcal{T}_{u n i}=\frac{\hbar}{k} \frac{1}{t_{u n i}}$, which is calculated in the eleventh column with the age in space, is extremely low and still falls with the age of the universe.

The twelfth column shows the variable gravitational value $\gamma$. It cannot be constant if the entropy of the universe is constant. It is calculated according to the formula: $\gamma=\frac{r_{u n i} c^{2}}{m_{u n i}}$.

In the thirteenth column, an important relationship is calculated which, like entropy, must remain constant: $\mathcal{T}_{\text {uni }}{ }^{3} V_{\text {uni }}=$ const.

The term in the fourteenth column is a measure of the entropy of the universe $\mathcal{T}_{\text {uni }} V_{\text {uni }}{ }^{x-1}=$ const. with a constant $x=4 / 3$.

The fifteenth column shows the entropy of the universe as a quotient of energy and temperature: $S_{\text {uni }}=\frac{E_{\text {uni }}}{\mathcal{T}_{\text {uni }}}$.

An alternative entropy calculation is shown in column 16 using formula (03). It can be seen that the entropy in columns 15 and 16 corresponds and remains constant.

It is interesting that with the age of space the whole Planck system must change completely with it: $\mathcal{T}_{\text {planck }} / \mathcal{T}_{\text {uni }}=m_{\text {uni }} / m_{\text {planck }}=t_{\text {uni }} / t_{\text {planck }}=r_{\text {uni }} / r_{\text {planck }}=8.110^{60}$. The following applies to the number of effects in the universe: $N=S_{\text {uni }} / k=6.510^{121}=\left(t_{\text {uni }} / t_{\text {planck }}\right)^{2}$. This number of effects is constant for all times.

\section{The principle of constant entropy}

Statistically, entropy indicates the number of microstates of a macrostate. For example, the equal distribution of equally sized gas molecules in a gas-tight and insulated container is the state with the greatest entropy. If there are on average more molecules in a certain area of the container, a balance in the direction of the uniform distribution takes place.

Each balancing process is time-dependent. The time can be included in the entropy description of objects. Time results from the structure of the mass, which must therefore decay for the formation of time [3.].

In information theory, entropy is also a measure for potential information [4.]. The maximum and constant entropy of the universe can be described as follows [5.]:

$$
S_{u n i}=\frac{k}{\hbar} m_{u n i} t_{u n i} c^{2}
$$

Here $m_{u n i}$ is the mass and $t_{u n i}$ the age of the universe. Since age is variable in the formula, the mass must fall with age if entropy is constant. Such a connection between time and entropy has been sought for a long time. This is due to the fact that in physical descriptions, time and entropy are regarded as quantities that can only change in one direction by themselves.

For an isentropic expansion there must also be an isentropic exponent. For space there are two important relationships with the temperature of the background radiation and the volume: $1 . \mathcal{T}_{b g}{ }^{3} V_{u n i}=$ const. and 2. $\mathcal{T}_{b g} V_{u n i}{ }^{x-1}=$ const. (see also Table 1). From this the value of $x=4 / 3$ can be calculated [1.].

\section{The mass}

The mass must therefore change according to equation (03) in such a way that the term $m_{\text {uni }} t_{\text {uni }}$ remains constant. This applies not only to the space mass, but also to any object mass $m_{x}$. A relationship in the form is known from quantum physics as energy-time uncertainty. In general, for a certain time period $\Delta t=$ $t_{\text {new }}-t_{\text {uni }}$ can be written with the new age $t_{\text {new }}$ for the new mass $m_{\text {new }}$ starting from an original mass $m_{0}$ : 


$$
m_{\text {new }}=\frac{m_{0}}{1+\frac{\Delta t}{t_{\text {uni }}}}
$$

This means, for example, that all masses were still twice as large at half the age of space.

The universe is becoming less energetic due to universal mass decay. The conservation of energy is of course still valid. In the course of mass decay, the gravitational energy of all objects increases. It becomes less negative and its amount in total is as large as the total mass energy:

$$
\sum E_{\text {object }}+\sum E_{\text {grav }}=0
$$

\section{The periods and the time}

With the age of the universe, the time base of all periodic processes must change. As the masses become smaller, the time base must become larger. For example, every photon becomes less energetic, its mass equivalent and frequency become smaller. In general, the periods increase with decreasing masses:

$$
T_{\text {new }}=T_{0}\left(1+\frac{\Delta t}{t_{u n i}}\right)
$$

Atomic clocks keep their resonance frequency constant, e.g. the caesium atomic clock keeps the frequency of 9,192,631,770 Hz. This corresponds to one second.

However, the frequency of all oscillations decreases with the mass decay, e.g. also the frequency of the atomic oscillation from $9,192,631,770 \mathrm{~Hz}$ to $9,192,631,769.33 \mathrm{~Hz}$ after one year. The regulator of the atomic clock, which adjusts the frequency of the microwave resonator according to the resonance frequency, now finds the maximum of the change of the energetic atomic states at this lower frequency. The second is now assigned to this lower frequency value, which is the new resonance frequency. If now for the distance measurement the running time of light is measured with this time scale, where one second is smaller than it should be, more seconds pass for the light path and a larger distance comes out. This distance is then actually as it is needed for distance measurement without time change.

In principle, one could count the oscillations of a second in a certain time interval and would come to the conclusion that with the age of the universe there are fewer oscillations.

If, as in nature, both the distance and all periods would increase with the age of the universe, the decayrelated distance growth would not be measurable at all. It is therefore an advantage for distance measurement that today's atomic clocks do not take the natural time base into account correctly. For example, a part of the annual distance growth earth-moon can be proven [6.].

\section{The distances, object sizes and wavelengths}

As the universe ages, all objects and distances increase in the same proportion. For example, the Bohr-radius of the hydrogen atom also increases. If one were to attempt to measure the phenomenon of distance increase with a scale ruler, one would not be able to determine the change, since the scale ruler has also increased. However, it is possible to determine the change in distance with a laser. Why this works is described in [6.].

The enlargement of all spatial distances, object dimensions and wavelengths is done with the same formula as for the periods:

$$
r_{\text {new }}=r_{0}\left(1+\frac{\Delta t}{t_{\text {uni }}}\right) \quad \lambda_{\text {new }}=\lambda_{0}\left(1+\frac{\Delta t}{t_{\text {uni }}}\right)
$$

Hereby the part of the above observed distance increase earth-moon, which is not tidal, and e.g. also the radius increase of the earth can be calculated.

\section{The temperature}

It is observed that the product of the third power of the temperature of the background radiation and the volume of space remains constant and $\mathcal{T}_{b g}{ }^{3} V_{u n i}=$ const. [7.]. Since the volume contains the radius in the third power, the following applies: $\mathcal{T}_{b g} r_{u n i}=$ const.

This relationship can also be generalized for any celestial objects that are not in further heat exchange with the environment: 


$$
\mathcal{T}_{\text {new }}=\frac{\mathcal{T}_{0}}{1+\frac{\Delta t}{t_{\text {uni }}}}
$$

As with the mass change, this means that the object temperatures were still twice as high at half the age of space.

\section{The gravitational constant}

Since, in order to maintain the structures and states of the objects described by physical laws, the ratio of electrical force and gravitational force must remain constant with space age, the correlation applies:

$$
\frac{F_{e l}}{F_{\text {grav }}}=\frac{e^{2} /\left(4 \pi \varepsilon_{0} r^{2}\right)}{\gamma m^{2} / r^{2}}=\text { const. }
$$

From this the principle $\gamma m^{2}=$ const. the development of the gravitational constant with space age can then be formulated as follows:

$$
\gamma_{\text {new }}=\gamma_{0}\left(1+\frac{\Delta t}{t_{\text {uni }}}\right)^{2}
$$

The gravitational value increases quadratically with time. Table 1 shows its development. With equation (10) it is easy to calculate that the increase over the period of a human life is extremely small and therefore not easy to observe. How does the gravitational force between two masses behave with the age of space? Because both masses fall with age, the gravitational constant increases quadratically with age, and the distance that increases with age and enters the equation quadratically, the gravitational force decreases quadratically with age. The gravitational field strength of a mass $\gamma m / r^{2}$ thus decreases linearly with the age of the universe.

\section{Relation to relativistic quantities}

Although the special theory of relativity is wrong [8.], a moving object is at rest with respect to the same object with a reduced velocity-dependent time lapse, an increased mass and a reduced object length in the direction of movement. Mass, period and length thus develop in exactly the opposite direction when energy is supplied (increase in velocity) than they do when the age of the universe increases.

Do the gravitational field strength and the temperature of the moving object also change when energy is supplied?

In order to maintain mathematical symmetry, these values must develop in exactly the opposite direction as they do with the reduction of energy that occurs with universal mass decay. This means that with the speed of the object, its gravitational field strength must increase and the object temperature must rise. The contraction in length leads to a structural compression, which results in an increase in temperature.

Thus the relativistic changes of the physical quantities take place in the opposite direction to the changes with space age. This is the only way to obtain a conclusive physical world view.

\section{The redshift of the galaxies}

With increasing distance the observed redshift increases. Of course, one observes the most distant galaxies in a past that is farthest back, since the time of light must be taken into account.

Related to a frequency measurement on the galaxies, which is performed with the help of an atomic clock, this means that the faulty frequency value, which is measured and compared with the oldest frequencies, also gives the largest error.

The known redshift $\mathrm{z}$ can be given in a wavelength or frequency dependence:

$$
z=\frac{\lambda_{o b s}}{\lambda_{0}}-1 \quad z=\frac{f_{0}}{f_{o b s}}-1
$$

Large masses have large gravitational fields and accordingly a large frequency shift of light in their near range. For the known gravitation-dependent redshift of a galaxy applies:

$$
z_{\text {grav }}=\sqrt{1-\frac{2 \gamma m_{\text {gal }}}{r_{\text {gal }} c^{2}}}-1
$$


It is interesting to note that the larger masses in the past have no influence on the measurement today, since the term under the root remains constant with space age (see 5., 7., 9.).

For the known velocity dependent redshift applies with velocity:

$$
z_{v}=\sqrt{\frac{c+v}{c-v}}-1 \quad \text { or converted to } v: \quad v=\frac{c z_{v}{ }^{2}+2 c z_{v}}{z_{v}{ }^{2}+2 z_{v}+2}
$$

As explained in point 6 , one second is assigned to a decreasing frequency value over time. Normally, like all periods, the second should increase over time, but it becomes smaller as the frequency decreases. All frequencies decrease with the age of the universe. Any accurate frequency measurement requires an accurate time base. The time base of an atomic clock (e.g. one second) is suitable for this purpose. If this time base changes, the measured frequency must also change. The observed frequency $f_{\text {obs }}$ of galaxies according to equation (11) is smaller than the original frequency $f_{0}$ when measuring the redshift. The further away the galaxy is, the older we perceive it and the higher the ratio $f_{0} / f_{\text {obs }}$ is observed. The observed frequency should actually be measured larger, since all frequencies used to be larger. On the other hand, it is measured smaller, because it is compared with the original frequency $f_{0}$, which, however, was determined during the frequency measurement with a too small time base and is therefore too large. This error also becomes larger with greater distances, since an even larger time base would have had to be used for the original frequency $f_{0}$ in order to correctly record its ever-smaller value. This leads to the misinterpretation of the galaxy velocities according to equation (13). Measuring means comparing. The measured value cannot be correct if the comparison value is wrong. The incorrectly determined redshift is interpreted as an increase in the speed of the galaxies with space. However, as explained in point 2, the speeds of the galaxies remain constant with the expansion of the universe. How big is the error?

The Hubble constant in SI units is $7.090410^{4} \frac{\mathrm{m}}{\mathrm{s}} / 3.085710^{22} \mathrm{~m}=2.297810^{-18} \frac{1}{\mathrm{~s}}$. Multiplied by the speed of light, the Hubble constant becomes an assumed acceleration: $a=6.888710^{-10} \mathrm{~m} / \mathrm{s}^{2}$. The expansion is to take place with this acceleration.

This is exactly the measurement error: The speed of light as the speed of the radius of space is related once too much to the age of space due to the incorrect measurement: $2.997910^{8} \frac{\mathrm{m}}{\mathrm{s}} / 4.352010^{17} \mathrm{~s}=$ $6.888710^{-10} \mathrm{~m} / \mathrm{s}^{2}$. However, no acceleration occurs, it has the value zero.

\section{Summary}

The expansion of the universe could be described as an isentropic system. The background radiation represents a distributed stellar radiation according to the above explanations. Some important physical quantities, such as the mass of the universe, the gravitational constant, as well as object dimensions and object distances, turn out to be variable. An accelerated expansion of the universe does not take place, the measured redshift is misinterpreted.

\section{References}

[1.] Gimsa, A., The beauty of nature, 2nd edition, p.70, Gieselmann Druck- und Medienhaus, Potsdam, Germany 2014, ISBN 978-3-923830-94-7.

[2.] Gimsa, A., The metric of space-time, English-German, p.73, published by the autor, Potsdam, Germany 2020, ISBN 978-3-00-064784-0.

[3.] See Source [2.], p.74-75.

[4.] Lyre, H., Informationstheorie, p.48, Wilhelm Fink Verlag München, Germany 2002, ISBN 3-82522289-6

[5.] See Source [1.], p. 42.

[6.] Gimsa, A., Development oft the Distance Earth-Moon, International Journal of Scientific Research and Management, Volume 08, Issue 03 March 2020, DOI: 10.18535/ijsrm/ v8i03.aa01.

[7.] Guth, A., Die Geburt des Kosmos aus dem Nichts, p.131, Die Theorie des inflationären Universums, Droemersche Verlagsanstalt Knaur München, Germany 2002, ISBN 3-426-77610-3.

[8.] Gimsa, A., The Phenomenon of Mass, International Journal of Scientific Research and Management, Volume 08, Issue 06 June 2020, DOI: 10.18535/ijsrm/v8i03.aa01. 